\title{
Rumor Dynamic Model considering Intentional Spreaders in Social Network
}

\author{
Yan Wang $\mathbb{D}^{1,2}$ Feng Qing $\mathbb{D},,^{1,2}$ and Lei Wang $\mathbb{D}^{3}$ \\ ${ }^{1}$ School of Data Science and Intelligent Media, Communication University of China, Beijing 100024, China \\ ${ }^{2}$ State Key Laboratory of Media Convergence and Communication, Communication University of China, Beijing 100024, China \\ ${ }^{3}$ Department of Mathematics, University of Manchester, Manchester, M13 9PL, UK
}

Correspondence should be addressed to Feng Qing; qing329364213@cuc.edu.cn

Received 13 November 2021; Accepted 4 February 2022; Published 26 February 2022

Academic Editor: A. E. Matouk

Copyright ( $\odot 2022$ Yan Wang et al. This is an open access article distributed under the Creative Commons Attribution License, which permits unrestricted use, distribution, and reproduction in any medium, provided the original work is properly cited.

\begin{abstract}
The development of network technology has created various platforms and methods for information dissemination. When rumors spread in social networks, they will rapidly spread and may cause social harm. Also, there are groups in social networks that create and spread rumors for the purpose of profit, thus expanding the scope of rumors. Therefore, based on the theory of complex network propagation dynamics, the study of the propagation law of rumors and the design of effective prevention and control strategies is of practical importance and theoretical significance for understanding the propagation laws of rumors and controlling the outbreak of rumors. The spreading process of rumors on social network platforms is focused here. The intentional spreader based on the classic rumor-spreading model is introduced. First, 2SIR rumor-spreading models on homogeneous and heterogeneous networks are established, respectively. Second, the steady-state analysis was separately carried out, and the corresponding propagation critical value was obtained: in the homogeneous network, the condition for the large-scale spread of rumors is $\alpha>m / \bar{k}$ or $\beta>\delta / \bar{k}$; in the heterogeneous network, the condition for the large-scale spread of rumors is $\alpha>m \bar{k} / \overline{k^{2}}$ or $\beta>\delta \bar{k} / \bar{k}^{2}$. Finally, the simulation calculation and model feasibility verification were carried out on the model. The results show that the theoretical propagation threshold corresponds with the simulation results. According to the simulation results, the final influence of rumors has significantly decreased with decreasing values of $\beta$ (intentional spreading rate) instead of $\alpha$ (unintentional spreading rate). It can be concluded that in the real-life rumor control process, more resources need to be invested in reducing the rate of intentional transmission instead of being indiscriminately put on controlling all spreaders of rumors.
\end{abstract}

\section{Introduction}

Rumors are news without fact, and their essential feature is unproven [1, 2]. "Webster English Dictionary" considers rumors to be a kind of gossip, rumor, or public opinion that lacks true evidence or is unproven, and it is difficult for the public to judge the authenticity. [3] Rumors can originate from people's fabrication of public events out of thin air based on real news but limited by objective conditions leading to incomplete information dissemination and so on. In most cases, the dissemination of rumors will cause a certain amount of harm to citizens and even the society. Small ones can damage personal credibility, and large ones can affect social stability and damage the image of the country. In the past, rumors could only be realized in the form of face-to-face communication, but with the development of social networks, the spread of rumors broke the limitations of time and space and can be widely spread in a short time. At the same time, the spreading path is becoming increasingly complex. In China, the social networking platforms commonly used by the public include Weibo, WeChat, etc., while abroad, there are Instagram, Twitter, and Facebook. The existence of these platforms has greatly reduced the cost of spreading rumors, and the impact has become more serious. Therefore, once a rumor is generated and spread on the internet, the public cannot easily distinguish the authenticity and may intentionally or unintentionally promote its spreading, making the situation out of control. 
The factors that affect the spread of rumors are the characteristics of the rumors themselves and the differences in individual behaviors. In reality, there is a phenomenon that individuals or organizations create "marketing accounts" for self-media marketing activities and participate in online public opinion events to drive traffic to achieve profitmaking purposes. The existence of such groups can easily magnify social phenomena [4], and in the process of spreading rumors, it is easy to give a great impetus to rumors. In addition, the individual behavior of the public also affects the direction of information dissemination. People's personality characteristics, herd psychology, trust in information, and discriminative ability are all important influential factors [5].

For most of the quantitative research on the spread of rumors, the epidemic model is the theoretical basis. In 1927, Kermack and McKendrick's SIR (susceptible-infected-recovered) model, SIS (susceptible-infected-susceptible) model, and threshold theory [6] were the earliest research on the dynamics of infectious diseases. Since the spread of rumors is highly similar to the epidemic, epidemic models are often used in quantitative research on rumor-spreading models. So, in the 1960s, Daley and Kendall [7, 8] first proposed using an infectious disease model to solve rumors in their paper, called the DK model. They divided the population into the following three categories: people who had not heard of rumors, people who spread rumors, and people who had heard but no longer spread rumors. These three groups correspond to "susceptible," "infected," and "recovered" in the SIR model, respectively. However, the key difference between the spread of rumors and the spread of the epidemic is that individuals' attitudes toward rumors are subjective, and the public has different ways of handling rumors under different educational backgrounds and social concepts. At the same time, the external environment of its propagation is also more complicated [9]. Therefore, in the research on the dynamics of rumor spreading, researchers have extended its direction to individual differences, complex networks, etc., to better simulate the real-spreading environment.

To minimize rumors' impact on public daily life and social stability, some mathematical model should be established to explore the law of rumor spreading. It is of practical significance to control the rumors in their spreading process.

In the application of the communication model, the SIR model and the SEIR (susceptible-exposed-infected-recovered) model are most widely used. In 2001, Zanette [10, 11] first combined the SIR model with complex network theory and proposed rumor dynamics on complex networks. Since then, the rumor-spreading dynamics on complex networks began to enrich. On the basis of Zanette's research, a large number of researchers consider the influence of many factors to improve their models.

Many researchers considered the differences in individual behavior and characteristics and introduced different states and behaviors into the model. Gu and Xia [12] proposed that if an individual in an immune state is an exposed state in the previous state, it may become a spreader with a certain probability after being exposed to rumors for a number of times and will not always maintain an immune state. Wang et al. [13] established a SIRaRu (spreader-ignorant-stifler1-stifler2) rumor-spreading model and studied the propagation and immune thresholds in homogeneous and heterogeneous networks, respectively. The mechanisms of forgetting and losing interest were introduced in the spread of rumors. It is found that the final size of rumors is greatly affected by the forgetting rate. Xia et al. [14] added the attractiveness and fuzziness of rumors to an improved SEIR rumor propagation model and introduced a hesitation mechanism in complex social networks. Huo et al. [15] established the SIbInIu (the four groups are recorded as S, Ib, In, and Iu, respectively) model and applied it to both homogeneous and heterogeneous networks. It is noteworthy that in the study, the author suggested that when a spreader contacts someone who has already known the rumor, the spreader will lose interest in spreading the rumor and will no longer propagate with probability. In addition, some researchers have classified spreaders. Huo et al. [16] considered the activity level of nodes and divided the types of spreaders into high frequency and low frequency, respectively, thus establishing an I2SR (ignorant-spreader1spreader2-stifler) model. Huo and Cheng [17] also considered the indiscernible degree, established an IWSR (ignorant-wiseman-spreader-stifler) model with a selfgrowth mechanism, and described the steady-state dynamic theory of rumor propagation by calculating the propagation threshold. Studies have shown that the stronger the individual's ability to grow, the smaller the scale of the rumors. At the same time, the greater indiscernible the degree of the rumors is, the greater the influence of the rumors will be.

In real life, for groups who do not believe in rumors, they may become the ones who hinder and prevent the spread of rumors. For this reason, Zan et al. [18] introduced the counterattack mechanism and resistance characteristics by adding the counterattack group $\mathrm{C}$ to the classical SIR model and introduced the SICR model to show the individual's selfresistance to rumors. Wan et al. [19] believed that in reality, besides the people who spread rumors, there are also people who spread the truth. Therefore, based on the SIR rumor propagation model, the SIERsEs (spreader-ignorant-eliminator-rstifler-estifler) model is built, and the rumor cleaner who will reveal the rumors by the truth is introduced into the model.

In addition, in social networks, there is not only one type of rumor spread at a time but also multiple rumors may spread at the same time. There may be a derivative or game relationship between these rumors. Liu et al. [20] proposed a susceptibility-hesitation-infection-removal (SHIR) model of dual information competition based on individual personalization and opinion divergence, in which the hesitation state is a neutral state in dual information competition. It is found that the final result of the competition is related to the ratio of two information communication thresholds. Trpevski et al. [21] extended the SIS model and established two rumor-spreading models with different acceptance probabilities, where one of the rumors will be prioritized and dominate the network. Wang [22] considered the high 
indiscernibility of rumors and the confrontation mechanism between rumors and truth in reality and proposed the 2SIH2R (spreader1-spreader2-ignorant-stifler1-stifler2) model. Finally, it is concluded that the higher the degree of indiscernibility, the greater the influence of rumors; the stronger the confrontation mechanism, the less the influence of rumors.

In summary, in recent years, research on rumorspreading models has become increasingly diversified. By subdividing various groups of people and considering the coexistence of multiple information, we can study communication behaviors that are more suitable for real social networks. So far, many works have contributed to the dynamic model of rumor spreading, but there are still some parts to be improved:

(a) In real life, the reasons why rumors can spread on a large scale can be roughly divided into two types: one of them is that rumors are highly indiscernible and closely related to daily life. Another reason is that some people intentionally spread rumors for benefits in the era of rapid development of social networks, which leads to the increasing influence of rumors. The abovementioned references only considered the influence of the rumors' indiscernibility in their model, but the influence of the people who intentionally spread rumors is not included.

(b) Second, most model topologies designed in references are linear serial structures, such as SIR or SEIR models. These models have low flexibility and versatility.

(c) Third, most previous works put all spreaders into one category, that is, these people are assumed to be not different. But in practice, people think differently when they spread rumors, and some people even do not even know they are spreading rumors.

(d) At last, most studies categorize people by considering that they are in different stages of rumor transmission, while seldomly dividing the people from people's motivation and purpose.

Therefore, we here consider the motivation-driven mechanism, model structure, spreader heterogeneity, and population classification of the rumor spreading to improve the existing models.

In reality, there is such a phenomenon that some individuals will unconsciously participate in the spread of rumors when they communicate with others on social networks. At the same time, there is also a phenomenon where the spread of online rumors is often further spread by intentional marketing or personal negative psychology. In the abovementioned references, this point is not considered. Based on the original SIR model, we here introduce the intentional spreaders to expand the model to a 2SIR (unintentional spreader-intentional spreader-ignorant-stifler) model and explore their influence in the spread of rumors. Then, the spreading threshold of the model in homogeneous and heterogeneous networks is studied and simulations are carried out to study the influence of changes in parameter values on the spread of the rumor.

\section{SIR Rumor Spreading Model}

In recent years, a large number of intentional spreading phenomena have appeared in the social network, which is a special situation in online spreading and has a strategic spreading effect. This type of spreading method is based on real information, by amplifying the content that is consistent with the audience's emotions to create rumors and attract attention.

In the era of pan media, the public lacks independent thinking on network events and has formed unconscious habits of disseminating information. Because of their curiosity, they are attracted by the mysterious events in a complex online environment, which drives the event to higher heat. In addition, some references have mentioned that information recipients have a higher degree of trust in the news of nonstakeholders such as family members, friends, and so on [23]. As a result, people will unconsciously become the promoter of rumor spreading, that is, unintentionally spreading rumors. Meanwhile, Shen [24] mentioned in the study that people pay more attention to the clout, freshness, and curiosity of social network information than the authenticity of the information. When people actively choose information, the overflow of negative information will weaken their judgment. Individuals with poor judgment are more susceptible to emotional arousal, especially by marketing-driven spreaders, which makes it easier for them to spread rumors.

However, even if rumors spread everywhere, the public will be willing to seek and explore the truth. Albert and Postman point out that the basic conditions for rumors to spread include the fuzziness of events [25]. When the fuzziness is smaller, that is, the public's revealing psychology is stronger, more details of the incident are dug up, and the influence of rumors will be reduced. Therefore, in recent models the parameter $m$ is introduced to represent the probability of the public seeking the truth.

In this section, we assume that people in the network can be divided into four categories: spreader $1\left(S_{1}\right)$, spreader 2 $\left(S_{2}\right)$, ignorant $(I)$, and stifler $(R)$. Among them, spreader 1 represents people who unintentionally spread the rumor; spreader 2 represents people who intentionally spread the rumor; ignorants are people who have never heard of the rumor; and stiflers are people who have heard the rumor but do not spread. The rumor-spreading process of the 2SIR is shown in Figure 1.

The rules of rumor spreading of the 2SIR model can be summarized as follows:

(a) When the ignorant contacts the unintentional spreader $S_{1}$, the ignorant converts to the unintentional spreader $S_{1}$ with probability $\alpha$ (unintentional spreading rate). When the ignorant contacts the intentional spreader $S_{2}$, the ignorant converts to the intentional spreader $S_{2}$ with probability $\beta$ (intentional spreading rate).

(b) When the unintentional spreader $S_{1}$ contacts the intentional spreader $S_{2}$, the $S_{1}$ may become $S_{2}$ with probability $\theta$. When the unintentional spreader $S_{1}$ 
contacts the people who have known the rumor $\left(S_{1}, S_{2}\right.$, and $\left.R\right)$, the former may become $R$ with probability $\lambda$ because of the loss of interest. Due to the influence of the external environment (e.g., media reports) and one's own education level, when the people in $S_{1}$ question the authenticity of a rumor, they will go to verify it and stop spreading it with probability $m$.

(c) When the intentional spreader $S_{2}$ loses the temptation of interest or the topic of interest declines over time, they become $R$ with probability $\delta$ and no longer spread the rumor.

In a homogeneous network, $I(t), S_{1}(t), S_{2}(t)$, and $R_{2}(t)$ represent the densities of the ignorant, spreader 1 , spreader 2 , and stifler, respectively, at time $t$. For any time $t$, there is always $I(t)+S_{1}(t)+S_{2}(t)+R(t)=1$. Based on the above rules of spreading of the 2SIR model, the mean-field equations can be given as follows:

$$
\begin{aligned}
& \frac{\mathrm{d} I(t)}{\mathrm{d} t}=-\alpha S_{1}(t) I(t) \bar{k}-\beta S_{2}(t) I(t) \bar{k} \\
& \frac{d S_{1}(t)}{\mathrm{d} t}=\alpha S_{1}(t) I(t) \bar{k}-\theta S_{1}(t) S_{2}(t) \bar{k}-\lambda S_{1}(t)\left(S_{1}(t)+S_{2}(t)+R(t)\right) \bar{k}-m S_{1}(t), \\
& \frac{d S_{2}(t)}{\mathrm{d} t}=\beta S_{2}(t) I(t) \bar{k}+\theta S_{1}(t) S_{2}(t) \bar{k}-\delta S_{2}(t), \\
& \frac{d R(t)}{\mathrm{d} t}=m S_{1}(t)+\delta S_{2}(t)+\lambda S_{1}(t)\left(S_{1}(t)+S_{2}(t)+R(t)\right) \bar{k}
\end{aligned}
$$

where $\langle k\rangle$ represents the average degree of the generated network.

Social media has become the main platform for network interaction and information dissemination due to its convenience, low cost, and speed. [26] It was found that in reality, many social networks are closer to heterogeneous networks, and the topological structure is more complex than homogeneous networks [27]. Therefore, studying the dynamic model of rumors on heterogeneous networks can more realistically simulate the spread of rumors in real networks. In a heterogeneous network, the mean-field equations can be given as follows:

$$
\begin{aligned}
\frac{d I_{k}(t)}{\mathrm{d} t}= & -\alpha k I_{k}(t) \sum_{k^{\prime}} S_{1 k^{\prime}}(t) P\left(k^{\prime} \mid k\right)-\beta k I_{k}(t) \sum_{k^{\prime}} S_{2 k^{\prime}}(t) P\left(k^{\prime} \mid k\right), \\
\frac{d S_{1 k}(t)}{\mathrm{d} t}= & \alpha k I_{k}(t) \sum_{k^{\prime}} S_{1 k^{\prime}}(t) P\left(k^{\prime} \mid k\right)-\theta k S_{1 k}(t) \sum_{k^{\prime}} S_{2 k^{\prime}}(t) P\left(k^{\prime} \mid k\right)-\lambda k S_{1 k}(t) \\
& \cdot \sum_{k^{\prime}}\left(S_{1 k^{\prime}}(t)+S_{2 k^{\prime}}(t)+R_{k^{\prime}}(t)\right) P\left(k^{\prime} \mid k\right)-m S_{1 k}(t), \\
\frac{d S_{2 k}(t)}{\mathrm{d} t}= & \beta k I_{k}(t) \sum_{k^{\prime}} S_{2 k^{\prime}}(t) P\left(k^{\prime} \mid k\right)+\theta k S_{1 k}(t) \sum_{k^{\prime}} S_{2 k^{\prime}}(t) P\left(k^{\prime} \mid k\right)-\delta S_{2 k}(t), \\
\frac{d R_{k}(t)}{\mathrm{d} t}= & m S_{1 k}(t)+\delta S_{2 k}(t)+\lambda k S_{1 k}(t) \sum_{k^{\prime}}\left(S_{1 k^{\prime}}(t)+S_{2 k^{\prime}}(t)+R_{k^{\prime}}(t)\right) P\left(k^{\prime} \mid k\right),
\end{aligned}
$$

where $I_{k}=N_{I, K} / N_{k}$ represents the number of the ignorants with degree $k$ divided by the total number of people with degree $k, P\left(k^{\prime} \mid k\right)$ is the probability that a node with degree $k$ is linked to one with degree $k^{\prime}$, and $\sum_{k^{\prime}} S_{1 k^{\prime}}(t) P\left(k^{\prime} \mid k\right)$ is the probability that an edge of a node with degree $k$ links to a spreader 1 node at time $t$.

\section{Steady-State Analysis}

In this section, through the steady-state analysis of the model, the conditions under which rumors can spread in a wide range of homogeneous and heterogeneous networks are obtained. In the early days of the spread of rumors, very 


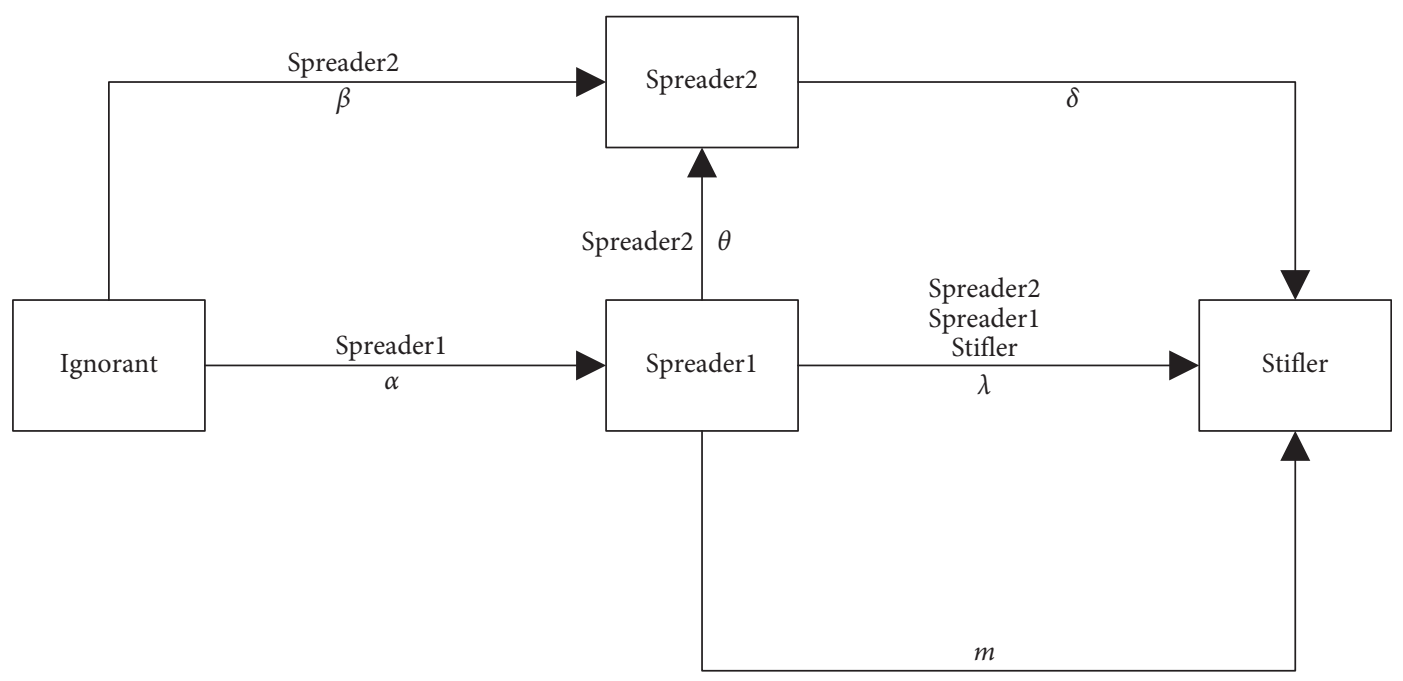

FIGURE 1: 2SIR rumor propagation model.

few people knew the rumors. It is assumed that there are $N$ nodes in the network. Therefore, without loss of generality, it is assumed that $I(0)=(N-2) / N \approx 1, S_{1}(0)=S_{2}(0)=1 /$
$N \approx 0$, and $R(0)=0$. In a homogeneous network, the Jacobian matrix corresponding to the systems (1)-(4) is as follows:

$$
J=\left[\begin{array}{cccr}
-\alpha S_{1}(t) I(t) \bar{k}-\beta S_{2}(t) I(t) \bar{k} & -\alpha I(t) \bar{k} & -\beta I(t) \bar{k} & 0 \\
\alpha S_{1}(t) \bar{k} & \alpha I(t) \bar{k}-\theta S_{1}(t) \bar{k}-\lambda(1-I(t)) \bar{k}-m & -\theta S_{1}(t) \bar{k} & 0 \\
\beta S_{2}(t) \bar{k} & \theta S_{2}(t) \bar{k} & \beta I(t) \bar{k}+\theta S_{1}(t) \bar{k}-\delta & 0 \\
0 & m+\lambda(1-I(t)) \bar{k} & \delta & 0
\end{array}\right] .
$$

Clearly, the systems (1)-(4) have a disease-free equilibrium $E_{0}=\left(I(0), S_{1}(0), S_{2}(0), R(0)\right)=(1,0,0,0)$. So, we have the following:

$$
J\left(E_{0}\right)=\left[\begin{array}{cccc}
0 & -\alpha \bar{k} & -\beta \bar{k} & 0 \\
0 & \alpha \bar{k}-m & 0 & 0 \\
0 & 0 & \beta \bar{k}-\delta & 0 \\
0 & m & \delta & 0
\end{array}\right] .
$$

The characteristic equation can be calculated as

$$
\left|J\left(E_{0}\right)-\mu E\right|=\left|\begin{array}{cccc}
-\mu & -\alpha \bar{k} & -\beta \bar{k} & 0 \\
0 & \alpha \bar{k}-m-\mu & 0 & 0 \\
0 & 0 & \beta \bar{k}-\delta-\mu & 0 \\
0 & m & \delta & -\mu
\end{array}\right|=0 .
$$

Solving equation (11), we can get the following: $\left(\mu_{1}, \mu_{2}, \mu_{3}, \mu_{4}\right)=(0,0, \alpha \bar{k}-m, \beta \bar{k}-\delta)$. According to the Routh-Hurwitz criterion, the system is stable at $E_{0}$ only when all eigenvalues are not greater than 0 , which means if the systems (1-4) are locally asymptotically stable at $E_{0}$, the condition that $\max \{\alpha \bar{k}-m, \beta \bar{k}-\delta\} \leq 0$ will be established. Moreover, the rumor cannot spread in a wide range, because the system is stable at the initial position. Thus, the condition for the rumor to spread on a large scale is $\alpha>m / \bar{k}$ or $\beta>\delta / \bar{k}$.

In a heterogeneous network, without loss of generality, it is assumed that $I_{k}(0)=(N-2) / N \approx 1$, $S_{1 k}(0)=S_{2 k}(0)=1 / N \approx 0$, and $R_{k}(0)=0$. According to $I_{k}=N_{k, i} / N_{k}$, it is easy to get $I_{k}(t)+S_{1 k}(t)+S_{2 k}(t)+R_{k}(t)=1$, which means if the number of three types of people is fixed, there is only one corresponding value of the remaining one. Therefore, research on systems (5)-(8) is equivalent to systems (5)-(7). Clearly, the systems (1)-(4) have a disease-free equilibrium $E_{1}=\left(I_{k}(0), S_{1 k}(0), S_{2 k}(0)\right)=(1,0,0)$. For convenience, we let $q\left(k^{\prime}\right)=P\left(k^{\prime} \mid k\right)=k^{\prime} P\left(k^{\prime}\right) / \bar{k}$.

The Jacobian matrix at $E_{1}$ corresponding to the systems (5)-(7) is as follows:

$$
J\left(E_{1}\right)=\left[\begin{array}{cccc}
A_{11} & A_{12} & \cdots & A_{1 n} \\
A_{21} & A_{22} & \cdots & A_{2 n} \\
\vdots & \vdots & \ddots & \vdots \\
A_{n 1} & A_{n 2} & \cdots & A_{n n}
\end{array}\right] \text {, where }
$$




$$
\begin{aligned}
A_{11} & =\left(\begin{array}{ccc}
0 & -\alpha q(1) & -\beta q(1) \\
0 & \alpha q(1)-m & 0 \\
0 & 0 & \beta q(1)-\delta
\end{array}\right), \\
A_{1 n} & =\left(\begin{array}{ccc}
0 & -\alpha \times 1 \times q(n) & -\beta \times 1 \times q(n) \\
0 & \alpha \times 1 \times q(n) & 0 \\
0 & 0 & \beta \times 1 \times q(n)
\end{array}\right), \\
A_{n 1} & =\left(\begin{array}{ccc}
0 & -\alpha \times n \times q(1) & -\beta \times n \times q(1) \\
0 & \alpha \times n \times q(1) & 0 \\
0 & 0 & \beta \times n \times q(1)
\end{array}\right), \\
A_{n n} & =\left(\begin{array}{ccc}
0 & -\alpha n q(n) & -\beta n q(n) \\
0 & \alpha n q(n)-m & 0 \\
0 & 0 & \beta n q(n)-\delta
\end{array}\right) .
\end{aligned}
$$

By the mathematical induction method, the characteristic equation can be calculated as follows:

$$
\mu^{n}(\mu+\mathrm{m})^{n-1}(\mu+\delta)^{n-1}\left(\mu^{2}+b \mu+c\right)=0,
$$

where $b=m+\delta-\alpha \sum_{k} k q_{k}-\beta \sum_{k} k q_{k}$ and $c=\left(\alpha \sum_{k} k q_{k}-m\right)$ $\left(\beta \sum_{k} k q_{k}-\delta\right)$.

Similar to the condition in a homogeneous network, it can be obtained that in a heterogeneous network, the condition for the spread of rumors is $\alpha>m \bar{k} / \overline{k^{2}}$ or $\beta>\delta \bar{k} / \overline{k^{2}}$.

\section{Numerical Simulation}

In this section, the Monte Carlo method is carried out in WS (Watts and Strogatz) small-world network [28] and BA (Barabási and Albert) scale-free network [29] to study the influence of parameter changes on various population trends. By referring to the simulation part of research [30-33], we set $N=100000$ in a WS small-world network with 8 edges per node, and the probability of random reconnection is 0.5 , setting $N=100000$ in a BA scale-free network, with 3 new edges per new node to the old nodes. Without special instructions, we set the following parameter values as follows: $\alpha=0.5, \beta=0.4, \delta=0.5, \lambda=0.1, \theta=0.2$, and $m=0.4$.

Figure 2 describes the process of density change over time in four populations: ignorant, spreader 1, spreader 2 , and stifler. As we can see, at the beginning of the model, the density of various populations is relatively stable, and there is no obvious change. After a period of time, the number of ignorant people rapidly drops, while the two types of spreaders rapidly rise and reach a peak. This shows that the rumor is spreading on the internet on a large scale. Then, the two types of spreaders begin to decline, and the model finally stabilizes. This shows that the rumors are gradually disappearing on the internet. From the comparison of the two networks, in the BA scale-free network, the overall evolution process of various groups of people in the process of spreading rumors is similar to that of the WS small-world network, but the time for the two types of spreaders to reach the peak is shorter than that in the WS small-world network. At the same time, it takes less time to reach a steady state.
Figure 3 shows the final density of the immunized person $R$ when the model is stable under different $\alpha$ and $\beta$ conditions to verify the spreading threshold of the model in the steady-state analysis part. Among them, the darker purple color represents the lower final density of the immune person $R$; the darker red color represents the greater final density of the immune person $R$. In the WS small-world network and BA scale-free network that have been set up, the average degree of WS small-world network is $\overline{k_{W S}}=8$; average degree of BA scale-free network is $\overline{k_{B A}}=5.999$ and $\overline{k_{B A}^{2}}=133.873$. Therefore, when $m=0.4$ and $\delta=0.5$ the theoretical propagation conditions of WS small-world network and BA scale-free network are $\alpha_{W S}>0.050$ or $\beta_{W S}>0.063$ and $\alpha_{B A}>0.018$ or $\beta_{B A}>0.022$, respectively. As shown in Figure 3(a), when $\alpha_{W S}>0.050$ or $\beta_{W S}>0.050$, the final number of immunized persons starts to be significantly nonzero. As shown in Figure 3(b), when $\alpha_{B A}>0.020$ or $\beta_{B A}>0.020$, the final number of immunized persons starts to be significantly nonzero. It can be seen that the error between the actual value and the theoretical value is very small and basically the same. It can also be seen from Figure 3 that the spreading threshold of the BA scale-free network is smaller. From this perspective, it can explain that the time for the two types of spreaders in Figure 2(b) to reach the peak is shorter than that in Figure 2(a). At the same time, it takes less time to reach a steady state.

Figure 4 describes the changes in the unintentional spreader over time under different $\alpha$ conditions. As shown in Figure 4, as $\alpha$ increases, the time for the unintentional spreader to reach the peak value is significantly shortened, and the peak is significantly increased, but the survival time of rumors will be significantly shortened. Under the same parameter conditions, the peak value of the unintentional spreader in the WS small-world network is higher than that of the BA scale-free network, but the time to reach the peak is longer.

Similarly, as shown in Figure 5, with the increase in $\beta$, the time for the intentional spreader to reach the peak is significantly shortened, and the peak is significantly increased, but the survival time of rumors will be significantly shortened. Under the same parameter conditions, the peak value of unintentional spreader in the WS small-world network is higher than that of the BA scale-free network, but the time to reach the peak is longer.

Figures 6 and 7, respectively, describe the maximum values of the two types of spreaders and the final density of immunized persons with $\alpha$ and $\beta$. As shown in Figure 6, with the increase in $\alpha$, the peak value of unintentional spreader gradually increases, and the influence of this type of spreader will also increase, while the peak value of intentional spreader will correspondingly decrease. However, the sum of the peaks of the two types of spreaders and the final number of stiflers did not significantly change.

Similarly, as shown in Figure 7, with the increase in $\beta$, the peak value of the intentional spreader gradually increases, and the influence of this type of spreader will also increase, while the peak value of the unintentional spreader will correspondingly decrease. The difference is 


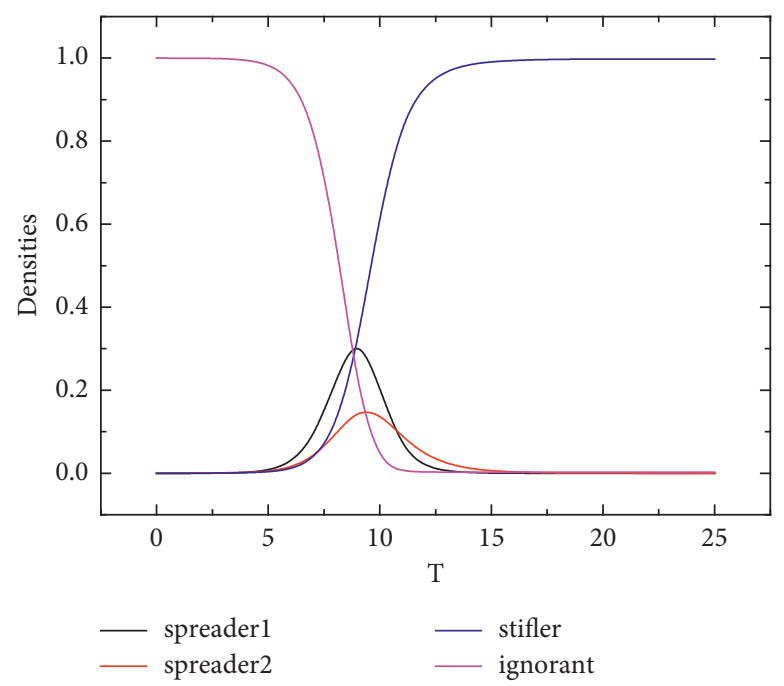

(a)

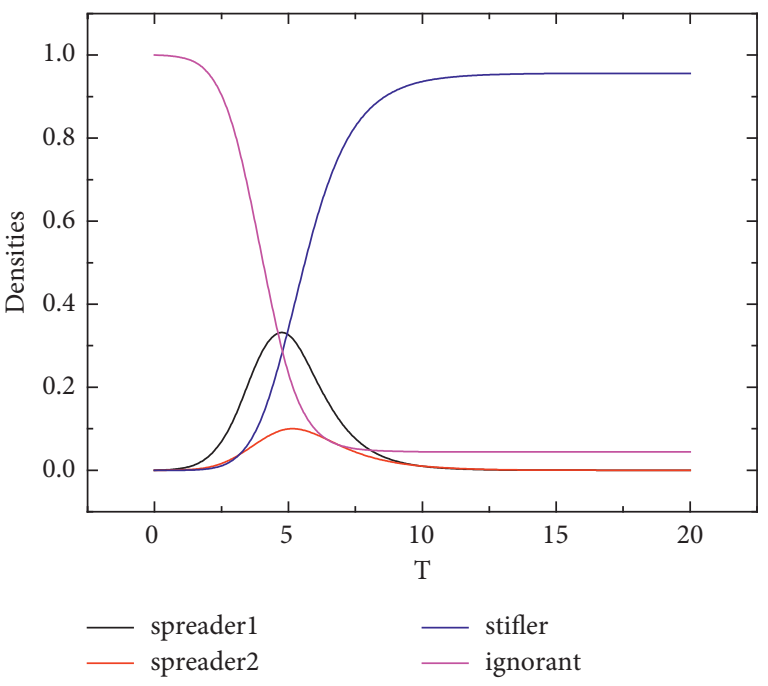

(b)

Figure 2: Density of four categories of people in different networks. (a) WS small-world network. (b) BA scale-free network.

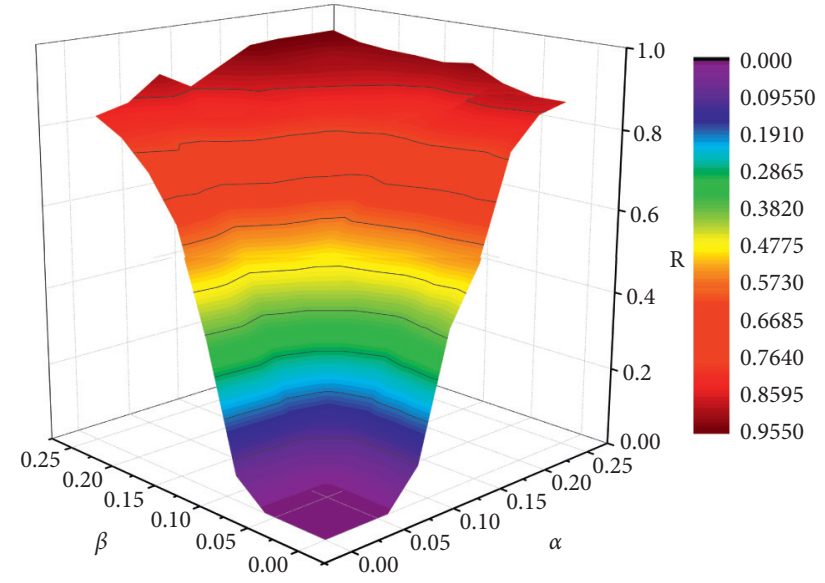

(a)

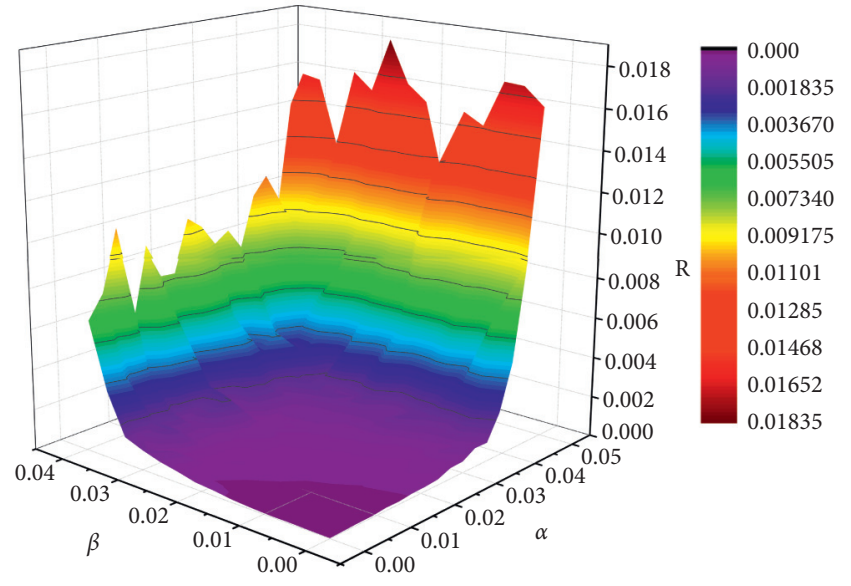

(b)

FIgURe 3: Density of stifler over time for different values of $\alpha$ and $\beta$ at steady state. (a) WS small-world network. (b) BA scale-free network.

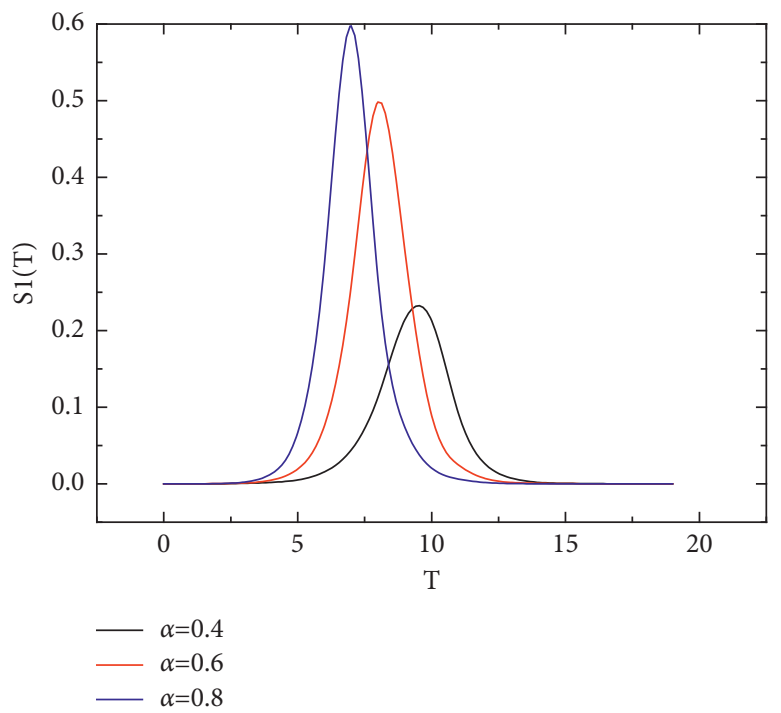

(a)

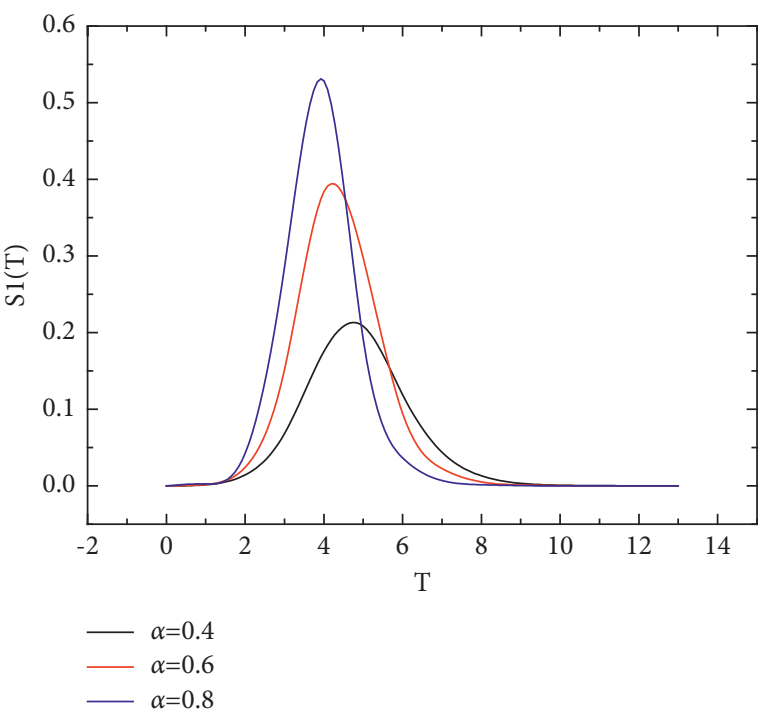

(b)

FIGURE 4: Density of spreader 1 over time for different values of $\alpha$. (a) WS small-world network. (b) BA scale-free network. 


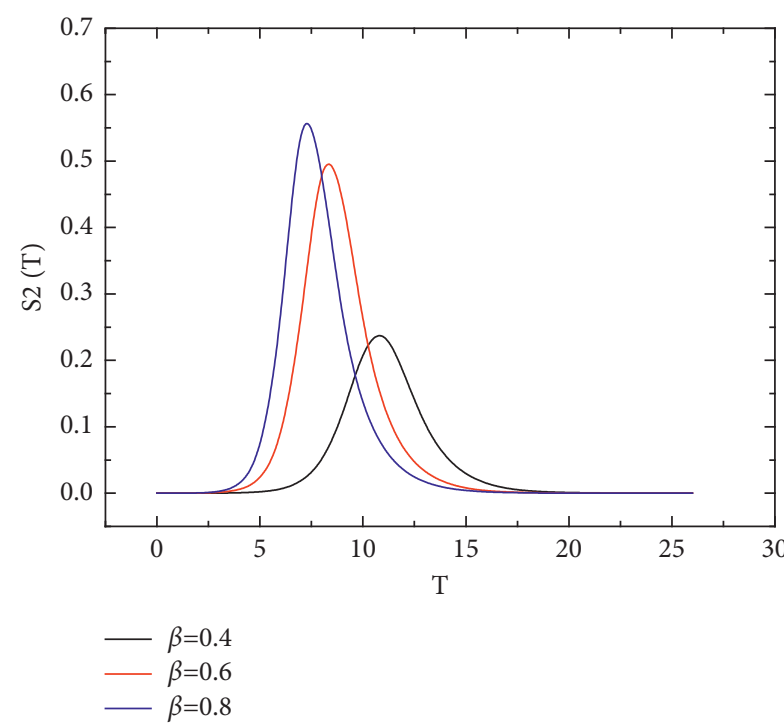

(a)

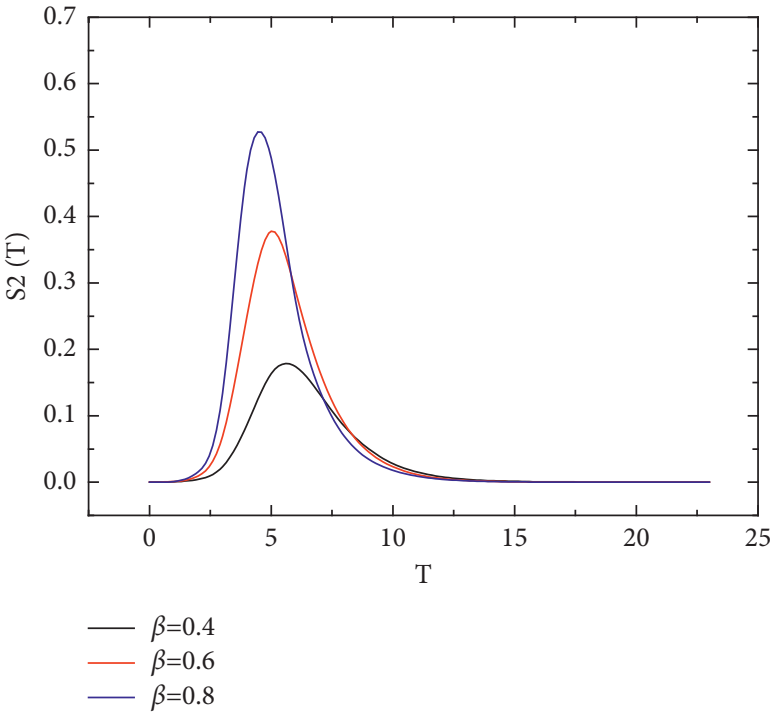

(b)

FIGURE 5: Density of spreader 2 over time for different values of $\beta$. (a) WS small-world network. (b) BA scale-free network.

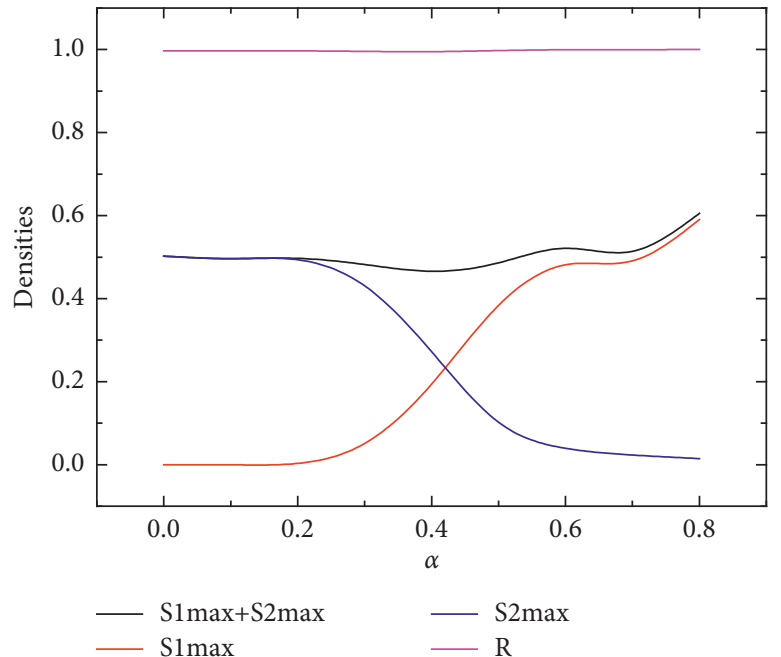

(a)

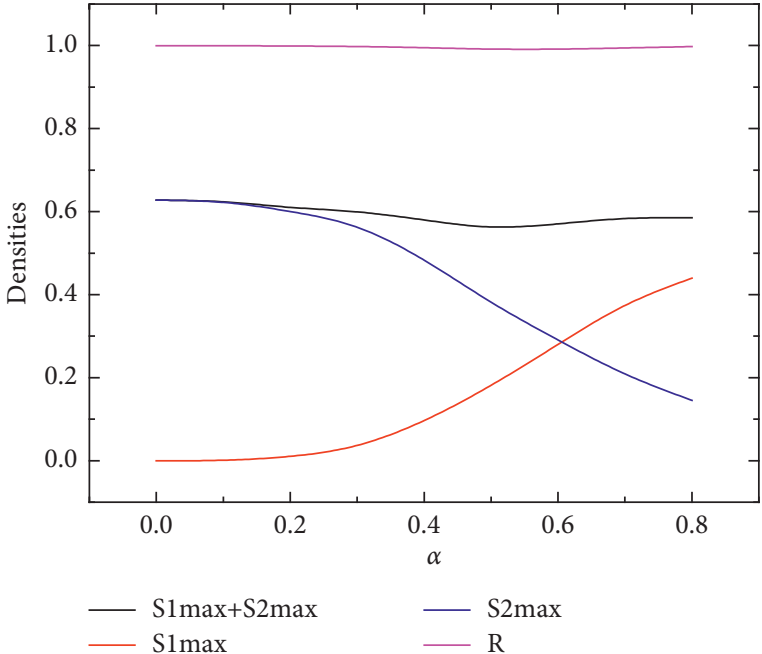

(b)

FIgURE 6: The influence of different values of $\alpha$ on rumor spreading. (a) WS small-world network. (b) BA scale-free network.

that the sum of the peaks of the two types of spreaders and the final number of immunized persons gradually rise to a stable value with the increase in $\beta$.
By comparing Figures 6 and 7, for the control of the rumor, more resources need to be devoted to reduce the intentional spreading rate $\beta$, rather than indiscriminately 


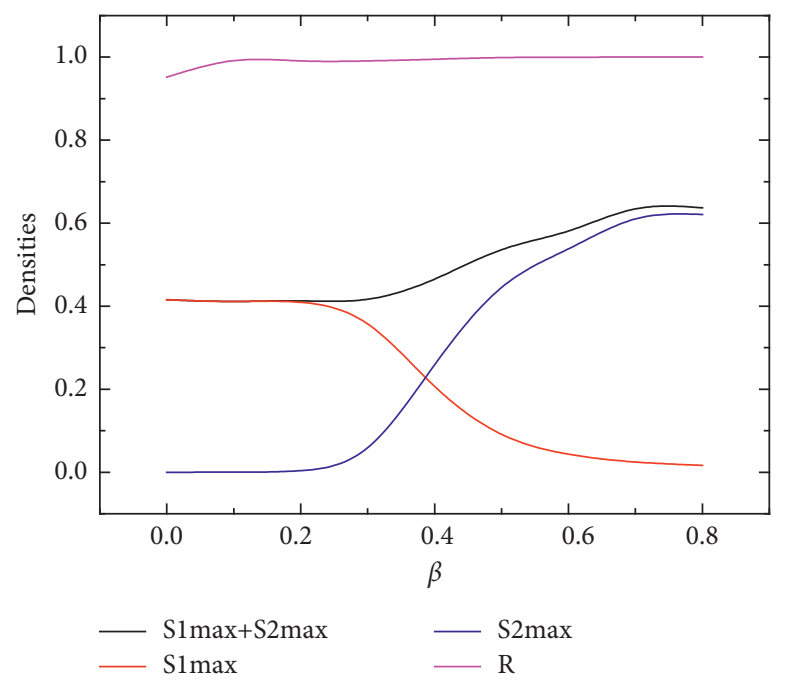

(a)

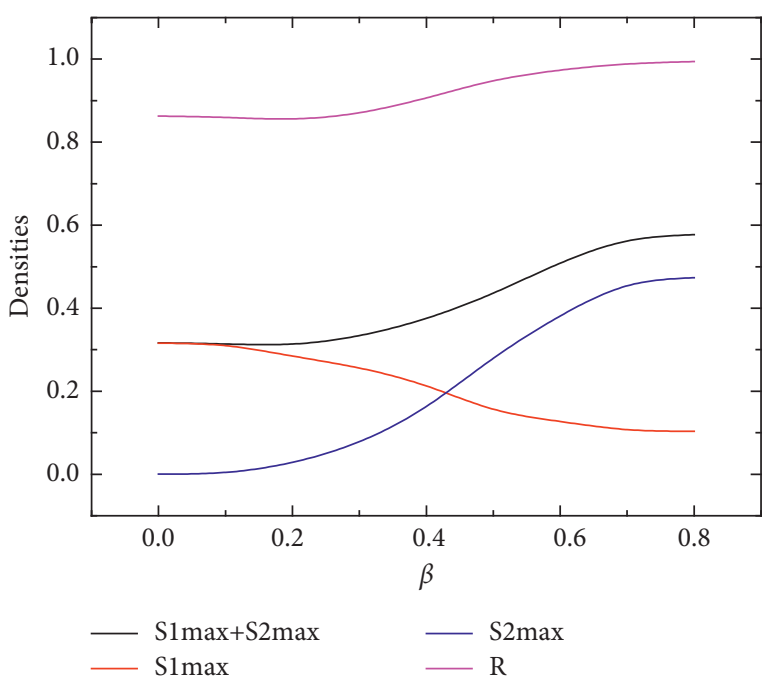

(b)

Figure 7: The influence of different values of $\beta$ on rumor spreading. (a) WS small-world network. (b) BA scale-free network.

investing resources to control all spreaders of rumors. As can be seen from Figure 6, as $\alpha$ decreases, the influence of intentional spread increases, and this is not desirable.

\section{Conclusions}

This study proposes a 2 SIR rumor-spreading dynamic model based on complex networks. The influence of intentional spreader on the spread of rumors is studied, and a differential dynamic system is established. The results through theoretical derivation of the spreading threshold and numerical simulation in Python are presented. In real life, due to the fast and wideranging characteristics of the internet, some individuals or organizations may gain attention and seek benefits by creating or participating in internet public opinion events. This type of spreader is the intentional spreader defined in this study. To study the influence of this group of people on the spread of the rumor, we introduce the second type of spreader on the basis of the classic rumor-spreading model and establish a 2SIR model. By deriving the dynamic equations, calculating the spreading threshold, and performing numerical simulation on the WS small-world network and the BA scale-free network, we get the following conclusions:

(1) For the 2SIR model, in the homogeneous network, the condition for the large-scale spread of rumors is $\alpha>m / \bar{k}$ or $\beta>\delta / \bar{k}$; in the heterogeneous network, the condition for the large-scale spread of rumors is $\alpha>m \bar{k} / \overline{k^{2}}$ or $\beta>\delta \bar{k} / \overline{k^{2}}$.

(2) In this study, since the spreading threshold of heterogeneous networks is smaller than that of homogeneous networks, the time for the two types of spreaders to reach the peak is shorter than in the homogeneous network, and at the same time, it takes less time to reach the steady state.

(3) With the increase in the two types of spreading rates, the time for the corresponding two types of spreaders to reach their peaks is significantly shortened, and the peaks are significantly increased, but the survival time of rumors will also be significantly shortened.

(4) The simulation results also show that reducing the unintentional spreading rate $\alpha$ does not significantly reduce the influence of rumors. In contrast, reducing the intentional spreading rate $\beta$ can obviously control rumors. It shows that in real life, more resources need to be devoted to reduce the intentional spreading rate, rather than indiscriminately investing resources on controlling all spreaders of rumors.

In the future, we will consider more influencing factors to improve our model, such as the influence of media and the competition between rumors and truth.

\section{Data Availability}

The generated data used to support the findings of this study have not been made available because the data are randomly generated according to the rules.

\section{Conflicts of Interest}

The authors declare that there are no conflicts of interest regarding the publication of this paper.

\section{Acknowledgments}

This paper was financially supported by the Beijing Municipal Natural Science Foundation (9202018), the Fundamental Research Funds for the Central Universities (CUC21GZ005, CUC2000004, CUC19ZD002, and 2018CUCTJ047), and the Academic Research Project of China Federation of Radio and Television Association (2020ZGLH015). 


\section{References}

[1] N. DiFonzo, N. M. Robinson, J. M. Suls, and C. Rini, "Rumors about cancer: content, sources, coping, transmission, and belief," Journal of Health Communication, vol. 17, no. 9, pp. 1099-1115, 2012.

[2] H. J. Oh and H. Lee, "When do people verify and share health rumors on social media? The effects of message importance, health anxiety, and health literacy," Journal of Health Communication, vol. 24, no. 11, pp. 837-847, 2019.

[3] N. Chao and X. Huang, "Research on "rumors" phenomenon in Internet communication," Information Studies:Theory \& Application, vol. 6, pp. 586-589+575, 2004.

[4] H. Yu and J. Li, "Prevention and treatment of enlarging public opinion risk from malicious media marketing," Journalism Lover, vol. 3, pp. 27-32, 2021.

[5] R. L. Rosnow, J. L. Esposito, and L. Gibney, "Factors influencing rumor spreading: replication and extension," Language \& Communication, vol. 8, no. 1, pp. 29-42, 1988.

[6] W. O. Kermack and A. G. A. Mckendrick, "A contribution to the mathematical theory of epidemics," Proceedings of The Royal Society A Mathematical Physical and Engineering Sciences, vol. 115, no. 722, pp. 700-721, 1927.

[7] D. J. Daley and D. G. Kendall, "Epidemics and rumours," Nature, vol. 204, no. 4963, p. 1118, 1964.

[8] D. J. Daley and D. G. Kendall, "Stochastic rumours," IMA Journal of Applied Mathematics, vol. 1, no. 1, pp. 42-55, 1965.

[9] L. Yang, Y. Feng, G. Hou, and W. Ni, "Research on crossplatform social network public opinion propagation model under the joint action of individual factors and external environment," Journal of Modern Information, vol. 41, no. 3, pp. 138-147+158, 2021.

[10] D. H. Zanette, "Critical behavior of propagation on smallworld networks," Physical review. E, Statistical, nonlinear, and soft matter physics, vol. 64, Article ID 050901, 2001.

[11] D. H. Zanette, "Dynamics of rumor propagation on smallworld networks," Physical review. E, Statistical, nonlinear, and soft matter physics, vol. 65, Article ID 041908, 2002.

[12] Y.-R. Gu and L.-L. Xia, "Propagation and inhibition of rumors in online social network," Acta Physica Sinica, vol. 61, no. 23, Article ID 238701, 2012.

[13] J. Wang, L. Zhao, and R. Huang, "SIRaRu rumor spreading model in complex networks," Physica A: Statistical Mechanics and Its Applications, vol. 398, no. 15, pp. 43-55, 2014.

[14] L.-L. Xia, G.-P. Jiang, B. Song, and Y.-R. Song, "Rumor spreading model considering hesitating mechanism in complex social networks," Physica A: Statistical Mechanics and Its Applications, vol. 437, no. 1, pp. 295-303, 2015.

[15] L. A. Huo, F. Ding, and Y. Cheng, "Dynamic analysis of a SIbInIu, rumor spreading model in complex social network," Physica A: Statistical Mechanics and Its Applications, vol. 523, no. 1, pp. 924-932, 2019.

[16] L. A. Huo, L. Wang, N. Song, C. Ma, and B. He, "Rumor spreading model considering the activity of spreaders in the homogeneous network," Physica A: Statistical Mechanics and Its Applications, vol. 468, no. 15, pp. 855-865, 2017.

[17] L. A. Huo and Y. Cheng, "Dynamical analysis of a IWSR rumor spreading model with considering the self-growth mechanism and indiscernible degree," Physica A: Statistical Mechanics and Its Applications, vol. 536, no. 15, Article ID 120940, 2019.

[18] Y. Zan, J. Wu, P. Li, and Q. Yu, "SICR rumor spreading model in complex networks: counterattack and self-resistance,"
Physica A: Statistical Mechanics and Its Applications, vol. 405, no. 1, pp. 159-170, 2014.

[19] Y.-P. Wan, D.-G. Zhang, and Q.-H. Ren, "Propagation and inhibition of online rumor with considering rumor elimination process," Acta Physica Sinica, vol. 64, no. 24, Article ID 240501, 2015.

[20] Y. Liu, S.-M. Diao, Y.-X. Zhu, and Q. Liu, "SHIR competitive information diffusion model for online social media," Physica A: Statistical Mechanics and Its Applications, vol. 461, no. 1, pp. 543-553, 2016.

[21] D. Trpevski, W. K. Tang, and L. Kocarev, "Model for rumor spreading over networks," Physical review. E, Statistical, nonlinear, and soft matter physics, vol. 81, Article ID 056102, 2010.

[22] Y. Wang, F. Qing, J.-P. Chai, and Y.-P. Ni, "Spreading dynamics of a 2 sih2r, rumor spreading model in the homogeneous network," Complexity, vol. 2021, Article ID 6693334, 9 pages, 2021.

[23] L. G. Schiffman and L. L. Kanuk, Consumer Behavior, Pearson Plc, London, UK, 2000.

[24] C. Shen, "Research on the conformity phenomenon of network news communication-comment on "network communication"," New Writing, vol. 7, p. 118, 2018.

[25] G. W. Allport and L. Postman, The Psychology of Rumor, Henry Holt and Company, New York, NY, USA, 1947.

[26] K. Shu, A. Sliva, S. Wang, J. Tang, and H. Liu, "Fake news detection on social media," ACM SIGKDD Explorations Newsletter, vol. 19, no. 1, pp. 22-36, 2017.

[27] L. Zhao, X. Qiu, X. Wang, and J. Wang, "Rumor spreading model considering forgetting and remembering mechanisms in inhomogeneous networks," Physica A: Statistical Mechanics and Its Applications, vol. 392, no. 4, pp. 987-994, 2013.

[28] D. J. Watts and S. H. Strogatz, "Collective dynamics of "smallworld” networks," Nature, vol. 393, no. 6684, pp. 440-442, 1998.

[29] A. L. Barabasi and R. Albert, "Emergence of scaling in random networks," Science (New York, N.Y.), vol. 286, no. 5439, pp. 509-512, 1999.

[30] A. I. E. Hosni, K. Li, and S. Ahmad, "Analysis of the impact of online social networks addiction on the propagation of rumors," Physica A, vol. 542, Article ID 123456, 2020.

[31] D. M. Fan, G. P. Jiang, Y. R. Song, and Y. Wei Li, "Novel fake news spreading model with similarity on PSO-based networks," Physica A, vol. 549, Article ID 124319, 2020.

[32] M. Yan, X. Lou, and Y. Wang, "Channel noise optimization of polar codes decoding based on a convolutional neural network," Wireless Communications and Mobile Computing, vol. 2021, Article ID 1434347, 10 pages, 2021.

[33] C. Y. Sang and S. G. Liao, "Modeling and simulation of information dissemination model considering user's awareness behavior in mobile social networks," Physica A, vol. 537, Article ID 122639, 2020. 